\title{
A Comparative Analysis of the Mechanical Role of Leaf Sheaths of Poaceae, Juncaceae, and Cyperaceae
}

\author{
Andreas Kempe, Martin Sommer, and Christoph Neinhuis \\ Department of Biology, Institute of Botany, Faculty of Science, Technische Universität Dresden, 01062 Dresden, Germany \\ Correspondence should be addressed to Andreas Kempe; andreas.kempe@tu-dresden.de
}

Received 16 January 2013; Accepted 22 March 2013

Academic Editor: Zed Rengel

Copyright ( 2013 Andreas Kempe et al. This is an open access article distributed under the Creative Commons Attribution License, which permits unrestricted use, distribution, and reproduction in any medium, provided the original work is properly cited.

\begin{abstract}
Similarities in structural organization of the culm in Poaceae, Juncaceae, and Cyperaceae such as leaf sheaths and the presence of intercalary meristems at every node suggest the same mechanical properties and, accordingly, the same functionality. Meristems are zones of tissue formation, which constitute areas of weakness along the entire culm and provide the basis for rapid shoot elongation. Leaf sheaths clasp the culm preventing the shoot from breaking, ensuring the rigidity to grow erectly and to avoid damage of the meristematic tissue. The mechanical influence of leaf sheaths was investigated in members of Poaceae, Juncaceae, and Cyperaceae in the flowering stage. Mechanical properties of Poa araratica, Bromus erectus, Arrhenatherum elatius (Poaceae), Luzula nivea (Juncaceae), and Carex arctata (Cyperaceae) were determined in three-point bending before and after the removal of leaf sheaths. The presence of leaf sheaths results in smoothing the distribution of flexural rigidity and therefore avoids stress peaks. The achieved maxima of relative contribution of leaf sheaths to entire flexural rigidity ranged from $55 \%$ up to $81 \%$ for Poaceae, $72 \%$ for C. arctata, and $40 \%$ for L. nivea. Across the investigated families, the mechanical role of leaf sheaths could be verified as essential for culm stability during development and beyond.
\end{abstract}

\section{Introduction}

One characteristic of the morphological feature of grass culms is the presence of intercalary meristems above every node. The growth is not restricted to one apical meristem; rather, the culm is able to grow at every internode $[1,2]$. Therefore, the culm is showing frequent alternations of weak, nonreinforced and stiff, fully developed tissues below and above the nodes particularly during development. The increased vertical growth speed, enabled through many areas of growth, comes at a cost. Additional stabilizing structures to reinforce the less stable meristems have to be provided. The leaf sheaths envelope the culm in the most fragile section avoiding possible damage by providing the crucial stiffness [3-5].

Culms of Poaceae without regard to their leaf sheaths are studied concerning flexural rigidity, Young's modulus, the relation between bending and torsional stiffness, and with regard to anatomy $[2,6-10]$. The mechanical significance of leaf sheaths especially for necessity in development of culms was shown for Arundo donax [11] and Miscanthus [12].
Zebrowski [5] paid special attention on this aspect in Triticale as well as Niklas [3,4] for Arundinaria tecta and Avena sativa. The contribution of leaf sheaths to the entire rigidity of the culm of Arundinaria tecta reached 33\% on average [4]. Previous work, in which Young's modulus and flexural rigidity of two cultivars of Avena sativa were measured, revealed an increasing Young's modulus of the culm over a period of two weeks before reaching the state of anthesis [3]. Interestingly, even after anthesis the leaf sheaths continue to play a significant mechanical function according to their higher second moment of area due to its geometric contribution to flexural rigidity. Similar results were obtained from Tiricale [5], in which even three weeks after anthesis the leaf sheaths still contribute significantly to flexural rigidity of the entire culm.

Although structurally very similar to grasses, mechanical properties of rushes (Juncaceae) or sedges (Cyperaceae) have not been studied in detail. Ennos [13] and Etnier [14] studied Cyperaceae, pointing out a characteristic triangular cross-section of culms. Neither biomechanical properties of Juncaceae nor a contribution of leaf sheaths to bending 
stiffness of Juncaceae and Cyperaceae have been characterized so far.

Our study aims at comparing the function of leaf sheaths in several grasses and grass-like plants from different families. In particular, the stabilizing role of leaf sheaths along the culm, as described for other Poaceae, will be addressed.

\section{Material and Methods}

For our study, five species from three families of the order of Poales were chosen to cover different plant heights: Poa araratica Trautv., Bromus erectus Huds., Arrhenatherum elatius (L.) P. Beauv. ex J. Presl \& K. Presl (Poaceae); Luzula nivea L. (DC) (Juncaceae); and Carex arctata Boott. from Cyperaceae. Luzula nivea, Carex arctata, and Poa araratica were taken from the Botanic Garden Dresden. Bromus erectus and Arrhenatherum elatius were available directly at the meadow in front of the Institute of Botany of the TU Dresden. Plants were harvested at full stage of anthesis, since shoot elongation stops at this point. B. erectus, C. arctata, and A. elatius represent tall grasses with culm lengths up to ca. $1.5 \mathrm{~m}$, while those of $P$. araratica in contrast reach up to $0.5 \mathrm{~m}$ in length and $L$. nivea up to $0.8 \mathrm{~m}$, respectively.

Flexural stiffnesses of stems were analysed in three-point bending tests using a standard testing machine (Zwick/Roell Z 2.5). Even though the risk of ovalisation and local buckling of the hollow culms at the supports of testing device is obvious, this method proved to be the most favourable compared to cantilever bending or Euler buckling tests [11]. In particular, for avoiding dehydration of fresh tissue while preparing and measuring, the fast and simple three-point bending method is best suited. In comparison to cantilever bending, it operates without embedding or clamping one end of the culm. Further, damage may occur directly at the meristematic zones when applying Euler buckling due to the highly anisotropic structure of culms and leaf sheaths, particularly considering open or overlapping leaf sheaths, may cause damage directly at the meristematic zones when applying Euler buckling.

The influence of leaf sheaths on flexural rigidity was investigated by measuring culm segments before and after removal of leaf sheaths in three-point bending. Only the leaf blades were removed before testing. In order to minimize the influence of shear, tests were carried out with a minimum span-to-depth ratio of 20 [15]. The specimens length was set between 40 and $70 \mathrm{~mm}$, and accordingly the distance between the supports ranged depending on species from $60 \mathrm{~mm}$ for A. elatius and $B$. erectus, $40 \mathrm{~mm}$ for $L$. nivea and C. arctata and $30 \mathrm{~mm}$ for P. araratica, respectively. The entire culm was divided in as much segments as possible. All stems were tested in fresh condition and used for testing within two hours after harvesting. The flexural rigidity was calculated from the slope of the linear regression of the applied bending force versus deflection. For further evaluation, the relative contribution of leaf sheaths to entire flexural rigidity is calculated by dividing flexural rigidity of the culm without leaf sheath through flexural rigidity of the culm with leaf sheath. The tests were performed quasistatically at a testing speed of $1 \mathrm{~mm} / \mathrm{min}$, reducing speed-dependent influences. Sources of errors are reduced by constant work conditions. Keeping the material in water prior to measurement reduced the influence of dehydration. However, biological materials characteristically show a broad variation of mechanical properties with respect to environmental conditions, for example, temperature, wind exposition, or soil nutrient content. The obtained data were evaluated by Kruskal-Wallis test with regard to differences of segments with and without leaf sheaths. $P$ values less than 0.05 were considered significant.

\section{Results}

For each species, six individuals were tested. All representatives of Poaceae possess four to five internodes. The lengths of the internodes increase while the overlap of leaf sheaths decreases towards the apex. The basal leaf sheaths clasped the culms of Poaceae over the whole internode length (Table 1). For all these plants, the absolute maximum leaf sheath lengths are found at the apical internode. In contrast, C. arctata has three to four and L. nivea six to seven internodes. Compared to the grasses, internode and leaf sheath lengths of $L$. nivea are rather constant, while in C. arctata the longest internode and leaf sheath are found above the basal one with a subsequent decrease towards the apex. The leaf sheaths of C. arctata extend over the entire basal internode. L. nivea does not show a leaf sheaths overlap at any internode as the other species do. $B$. erectus, C. arctata, and $L$. nivea have closed leaf sheaths, whereas the others two species have open leaf sheaths.

The values for flexural rigidity obtained for Poaceae are very similar. The leaf sheath's function as stiffening structure has been lost or is only minimal in the basal internodes (leaf sheaths occasionally are dried and fall off), while it increases towards the apical internode. There, the relative contribution of leaf sheaths to entire flexural rigidity is a maximum and contributes significantly to the entire rigidity of the internodes (Table 1). The relative contributions range on average from $55( \pm 10) \%$ for A. elatius and about $76( \pm 8) \%$ for $P$. araratica to $81( \pm 18) \%$ for B. erectus (Table 1$)$.

In contrast, the leaf sheaths of C. arctata account for 66 $( \pm 14) \%$ to $72( \pm 26) \%$ of the flexural rigidity at the meristematic zones of all internodes (Table 1). The leaf sheath's contribution to flexural rigidity of $L$. nivea equally reaches ca. $40( \pm 11) \%$ in the three apical internodes while decreasing towards the base (Table 1). All species exhibit a high natural variability, which most probably arises from environmental influences.

Representative gradients of flexural rigidity for each species depending on distance from base and position within internodes are shown in Figure 1. The rigidity of culms including leaf sheaths (black dots) is always higher than that of the culms lacking leaf sheaths (circles); that is, leaf sheaths increase the rigidity of the culm segment which they cover (Table 2). The limited length of leaf sheaths did not always allow the measuring for more than one segment per internode ( $B$. erectus, $P$. araratica, and $L$. nivea). In these cases, it was not possible to detect a gradient (Figures 1(b), 1(c), and $1(\mathrm{~d}))$. In A. elatius and C. arctata, however, 
TABLE 1: Internode length, overlap of leaf sheaths, and relative contribution of leaf sheath to flexural rigidity of entire culm of all species. Standard deviation is bracketed and expressed as a percentage. Asterisks denote significant differences (Kruskal-Wallis test) between the segment below the node and the first segment above the meristem with and without leaf sheaths.

\begin{tabular}{|c|c|c|c|c|c|}
\hline & A. elatius & B. erectus & P. araratica & L. nivea & C. arctata \\
\hline Culm length [mm] & $1398(126)$ & $1053(109)$ & $467(29)$ & $683(54)$ & $980(74)$ \\
\hline \multirow[t]{2}{*}{ Number of internodes } & 5 & $4-5$ & 5 & $6-7$ & $3-4$ \\
\hline & \multicolumn{5}{|c|}{ Internode length (\% of entire length) } \\
\hline \multicolumn{6}{|l|}{ Internode } \\
\hline 1 (base) & $6.3(3.3)$ & $3.2(1.1)$ & $5.0(2.3)$ & $9.8(5.9)$ & $10.4(5.7)$ \\
\hline 2 & $12.0(3.2)$ & $8.5(3.8)$ & $10.7(1.4)$ & $14.1(3.3)$ & $41.1(3.0)$ \\
\hline 3 & $16.1(0.9)$ & $17.2(4.9)$ & $14.5(0.6)$ & $16.5(1.8)$ & $32.4(4.5)$ \\
\hline 4 & $21.3(2.0)$ & $30.3(5.9)$ & $21.5(1.3)$ & $16.5(1.8)$ & $16.2(1.8)$ \\
\hline 5 & $44.3(6.9)$ & $40.8(9.1)$ & $44.0(1.5)$ & $16.0(1.3)$ & \\
\hline 6 & & & & $15.9(2.5)$ & \\
\hline \multirow[t]{2}{*}{7} & & & & $11.1(7.8)$ & \\
\hline & \multicolumn{5}{|c|}{ Overlap of leaf sheaths (\%) } \\
\hline \multicolumn{6}{|l|}{ Internode } \\
\hline 1 (base) & 111.1 & $181.5(38.5)$ & $122.0(23.3)$ & $66.9(10.4)$ & $143.3(90.3)$ \\
\hline 2 & $84.4(15.9)$ & $111.1(53.4)$ & $78.2(4.5)$ & $56.6(13.1)$ & 27.5 (1.7) \\
\hline 3 & $72.8(9.4)$ & $62.4(15.5)$ & $63.9(3.6)$ & $41.9(7.0)$ & $24.7(6.5)$ \\
\hline 4 & $66.7(8.3)$ & $41.9(5.0)$ & $44.4(3.4)$ & $38.0(4.5)$ & $41.4(13.5)$ \\
\hline 5 & $45.2(7.1)$ & $34.1(4.3)$ & $22.6(1.3)$ & $36.2(4.4)$ & \\
\hline 6 & & & & $33.2(5.8)$ & \\
\hline 7 & & & & $49.8(25.6)$ & \\
\hline
\end{tabular}

$\begin{array}{lc}\text { Internode } & - \\ 1 \text { (base) } & - \\ 2 & 11.1(9.5) \\ 3 & 26.8(16.4) \\ 4 & 54.9(10.5)^{*} \\ 5 & \\ 6 & \\ 7 & \end{array}$

Relative contribution of leaf sheath to flexural rigidity of entire culm (\%)

$\begin{array}{cccc}- & 10.3 & 5.64 & 71.9(2.8) \\ - & 12.2(9.9) & 6.2(4.7) & 72.3(26.0)^{*} \\ 33.6(8.1) & 21.8(7.3) & 11.0(9.8)^{*} & 71.7(19.4)^{*} \\ 55.7(24.8)^{*} & 30.2(9.2)^{*} & 21.3(10.8)^{*} & 65.9(14.2) \\ 81.3(18.1)^{*} & 76.3(7.6)^{*} & 39.9(10.6)^{*} & \\ & & 40.0(5.9)^{*} & \\ & & 37.2(11.9)^{*} & \end{array}$

the internode could be subdivided revealing that the contribution of leaf sheaths to flexural rigidity decreases with increasing distance from the meristem. Further on, A. elatius, $B$. erectus, and $C$. arctata lose the basal leaf sheaths, and the rigidity of the culms exhibits higher values compared to the apex (e.g., A. elatius $1.9 \mathrm{Nm}^{2}( \pm 0.4)$ at base and $0.5 \mathrm{Nm}^{2}( \pm 0.2)$ at apex, see Table 2). Despite high rigidity of basal internodes in $P$. araratica and $L$. nivea, the leaf sheaths are still present and further increase the entire rigidity, although only to a low amount. A steep increase of the culm's rigidity along the internode in apical direction (2nd and 3rd internodes of $B$. erectus and C. arctata) shows the development from fresh unreinforced tissue in active meristems into fully differentiated tissue. Small or hardly recognisable gradients consequently are observed in mature culms (from 1st up to 4 th internode of $A$. elatius and $P$. araratica).

\section{Discussion}

Culms of grasses and grass-like plants possess an extraordinary slenderness ratio. The evolutionary benefit is to gain a maximum height of the inflorescences and thus of the infructescences, which guarantees a greater distance for seeds to be dispersed $[10,13]$. However, that architecture exhibits some risks with regard to mechanical stability. With a high slenderness ratio, the grass culms are at risk of buckling due to wind load and own weight reducing the success of dispersal $[3,4]$. Thus, the plants have evolved certain strategies in order to survive successfully. One method to handle height and stability is supported growth in a dense stand, for example, described for cereals $[7,10]$. In absence of supporting plants, the height is restricted by the response of plants to environmental influences [7]. Another characteristic is the multiple growth in each of the intercalaryy meristems that account for rapid shoot elongation. For this reason grasses, and grass-like plants typically exhibit abrupt transitions from fully developed stiff to weak meristematic tissues within a short distance at every node. In agreement with Niklas $[3,4]$, Spatz et al. [11], and Zebrowski [5], we found that the young culms have a low flexural rigidity near the meristem above the node, whereas the culm below the node reaches full rigidity. This mechanical weak point is compensated by leaf 


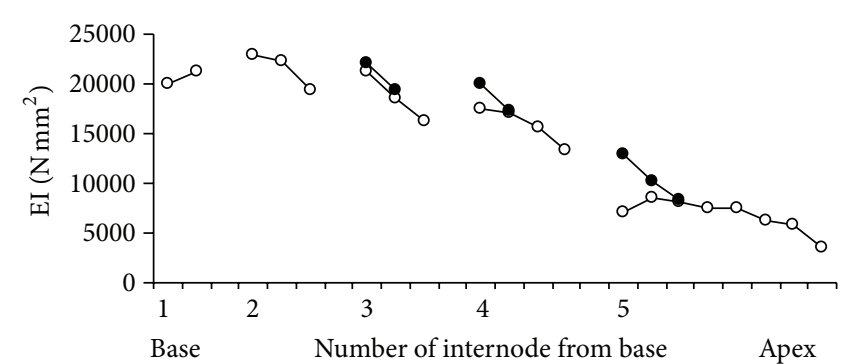

(a)

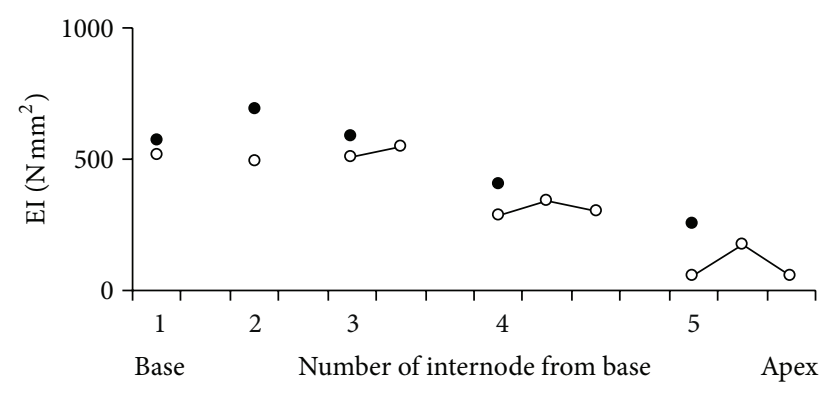

(c)

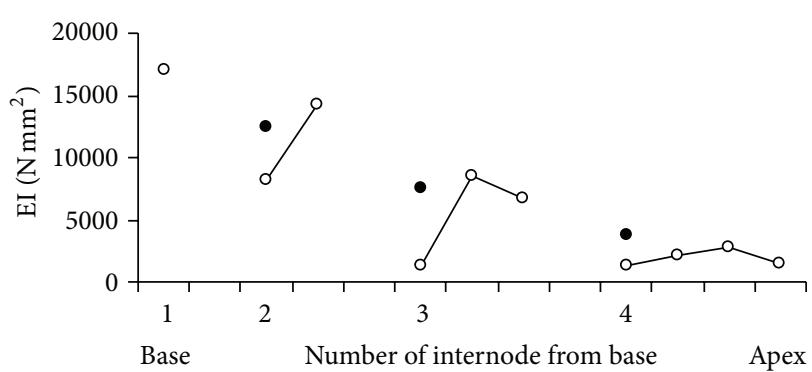

(b)

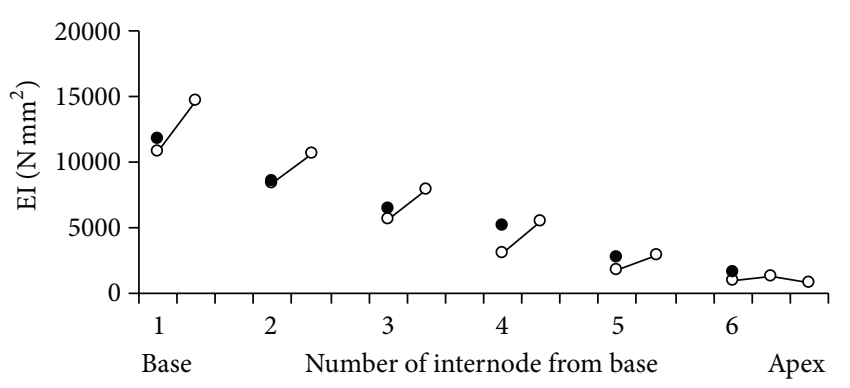

(d)

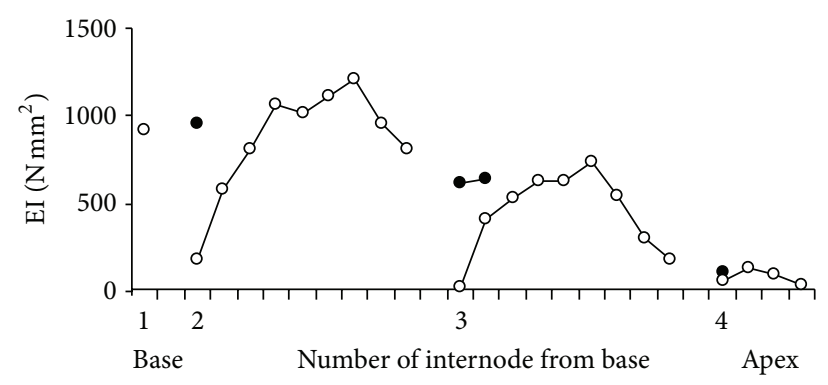

(e)

FIGURE 1: Representative gradients of flexural rigidity for individuals of (a) A. elatius (plant height $h=1473 \mathrm{~mm}$ ), (b) B. erectus ( $h=1071 \mathrm{~mm}$ ), (c) P. araratica $(h=509 \mathrm{~mm})$, (d) L. nivea $(h=662 \mathrm{~mm})$, and (e) C. $\operatorname{arctata}(h=1046 \mathrm{~mm})$ with $(\bullet)$ and without $(\mathrm{o})$ leaf sheath. Lines are interrupted where nodes are located.

sheaths, which are able to provide additional rigidity. In our investigations, the achieved maxima of relative contribution of leaf sheaths to entire flexural rigidity ranged from about $81 \%$ for B. erectus, $76 \%$ for P. araratica, $72 \%$ for C. arctata, and $55 \%$ for A. elatius to $40 \%$ for L. nivea. These data are in a same range as, for example, from Niklas [4] (33\% relative contribution) or Zebrowski [5] (almost 100\% relative contribution next to the meristem). Consequently, risk of failure due to local weakness is reduced by the presence of stiff leaf sheaths. The highly varying flexural rigidity along the plant axis of the leafless culm gets smoothed through the stabilization by leaf sheaths. We found this relationship to be ubiquitous, independent of plant heights, internode, and leaf sheath lengths, or differing in meristematic activity. Once the culm development is completed, high overall rigidity depends to a lesser extent on leaf sheaths, as shown for some basal internodes. For example, the achieved minima of relative contribution of leaf sheaths ranged from $6 \%$ for L. nivea. to $10 \%$ for $P$. araratica. A. elatius and B. erectus shed their basal leaf sheaths. Our studies have shown that all investigated species depend on leaf sheaths for protection and supporting of the mechanical weak points that occur during development of the culm. The relative contribution of leaf sheaths to entire flexural rigidity not only differs between the species but also depends on the position of the internodes within the culm as well as on the growth stages. For Poaceae, these contributions are distinctly higher towards the apex than towards the base. We also found that the zones of high relative contribution to flexural rigidity represent zones of greater meristematic activity. But the contribution may also remain on fully developed culms and still support the fully developed plant (e.g., in base of $P$. araratica). Differences due to open or closed leaf sheaths are not identifiable.

Which is new now relating to the representatives of rushes and sedges? C. arctata exhibits a flexural rigidity of nearly zero next to the meristem of every node, and the contribution of the leaf sheath is high at these positions. Consequently, the meristems may be active along the entire stem at anthesis, in contrast to the Poaceae. Anatomical differences between $C$. arctata and the grasses are a smaller overlap of leaf sheaths, without influence on contribution to entire stability close to the meristem. But the gradient of flexural rigidity along 
TABLE 2: Mean of flexural rigidity of entire internodes with and without leaf sheaths. Standard deviation is bracketed and expressed as a percentage.

\begin{tabular}{|c|c|c|c|c|c|}
\hline & A. elatius & B. erectus & P. araratica & L. nivea & C. arctata \\
\hline & \multicolumn{5}{|c|}{ Flexural rigidity of entire internodes without leaf sheaths $\left(\mathrm{Nm}^{2}\right)$} \\
\hline \multicolumn{6}{|l|}{ Internode } \\
\hline 1 (base) & $1.92(19.2)$ & $1.31(14.5)$ & 0.05 & 1.43 & $0.08(56.0)$ \\
\hline 2 & $1.70(25.9)$ & $2.33(67.5)$ & $0.05(10.3)$ & $1.20(29.4)$ & $0.08(39.7)$ \\
\hline 3 & $1.26(34.1)$ & $1.16(61.2)$ & $0.05(11.0)$ & $0.78(37.1)$ & $0.03(60.0)$ \\
\hline 4 & $0.92(43.7)$ & $0.62(75.9)$ & $0.03(11.8)$ & $0.50(39.3)$ & $0.01(52.0)$ \\
\hline 5 & $0.49(42.9)$ & $0.28(34.9)$ & $0.01(67.5)$ & $0.28(50.0)$ & \\
\hline 6 & & & & $0.17(50.1)$ & \\
\hline \multirow[t]{2}{*}{7} & & & & $0.07(45.3)$ & \\
\hline & \multicolumn{5}{|c|}{ Flexural rigidity of entire internodes with leaf sheaths $\left(\mathrm{Nm}^{2}\right)$} \\
\hline \multicolumn{6}{|l|}{ Internode } \\
\hline 1 (base) & - & - & 0.06 & 1.35 & $0.09(32.6)$ \\
\hline 2 & - & - & 0.06 & $1.01(30.8)$ & 0.08 \\
\hline 3 & $1.27(42.2)$ & $1.28(41.2)$ & $0.06(5.5)$ & $0.77(32.2)$ & $0.06(32.3)$ \\
\hline 4 & $1.01(43.3)$ & $0.79(32.2)$ & $0.04(6.5)$ & $0.51(38.7)$ & $0.03(45.8)$ \\
\hline 5 & $0.65(41.2)$ & $0.42(8.1)$ & $0.02(18.2)$ & $0.34(45.2)$ & \\
\hline 6 & & & & $0.20(45.8)$ & \\
\hline 7 & & & & $0.10(44.5)$ & \\
\hline
\end{tabular}

the stem seems less smoothed (Figure 1). Pointing out the overall rigidity of $C$. arctata is about one order of magnitude lower than the Poaceae of same height (resp. in the order of the much smaller $P$. araratica); the smoothing character is appropriate.

Every species has its individual gradient. L. nivea with more short internodes, relative short leaf sheaths, and small leaf sheath overlap shows a lower contribution of leaf sheaths to the entire flexural rigidity than the other investigated species. Particularly, in basal internodes the smoothing character of leaf sheaths is low. Nevertheless, leaf sheaths of $L$. nivea persist still at the base and contribute to all internodes rigidity during anthesis such as $P$. araratica. These results confirm an equal functional morphology of Cyperaceae and Juncaceae as was found for Poaceae and are in agreement with earlier studies.

However, all these results denote only a snapshot during development of fast-changing material properties. Therefore, the demonstrated differences between the species may be a result of different systematic groups (Cyperaceae, Juncaceae, and Poaceae) or due to slightly different ontogenetic stages.

Summing up, whereas the leaf sheaths exhibit full flexural rigidity at the meristem, the rigidity of the culm shows minimal flexural rigidity at the meristem which then increases towards the following node. The maximum contribution of leaf sheaths to flexural rigidity is supposed to occur during the growth of the culm, when the activity of the meristems is high.

Furthermore, failure of the culm is reduced by the leaf sheath construction, which reduces ovalisation [3]. Inspired by the mechanical role of leaf sheaths in rattan palms [16], another great benefit of grasses may be obvious. Under excessive bending, leaf sheaths may break at first. Assuming the enveloped culm still has the required flexibility, it bends easily and remains intact. Its flexibility ensures the survival of the culm and provides the possibility to reorientate for further growth. It could be observed that, before anthesis, lodged cereals are capable to reerect the upper internodes $[17,18]$. The loss of the leaf sheath is a programmed and calculable investment for the plant. After full development of culm and reduced contribution of leaf sheaths to flexural rigidity, the stability contribution by leaf sheaths is negligible and the necessity of fail-safe properties becomes dispensable when the maturity ends, for example, in the basal internodes of Poaceae. This leaf-sheath-culm construction contains a failsafe ability to survive break damage and consequently the possibility to reerect itself during elongation.

In summary, we showed the following.

(1) Leaf sheaths contribute significantly to the flexural rigidity of the entire culm at flowering stage.

(2) Alternating rigidity gradients within the whole culm length were compensated by the leaf sheaths.

(3) Leaf sheaths' contribution to flexural rigidity were found to be crucial for stability in the species of Poaceae as well as to the studied species of Juncaceae and Cyperaceae.

\section{Acknowledgments}

This work was financed by the European Union and the Free State of Saxony (ECEMP E2 - 13927/2379). The authors thank the Botanic Garden Dresden for providing them with plants. They acknowledge Sandrine Isnard, Lena Frenzke, Marko Storch, and Hanns-Christof Spatz for helpful discussions and constructive advices. 


\section{References}

[1] K. J. Niklas, "Plant height and the properties of some herbaceous stems," Annals of Botany, vol. 75, no. 2, pp. 133-142, 1995.

[2] H. Prat, "Recherches sur la structure et le mode de croissance des chaumes," Annales des Sciences Naturelles Botanique, vol. 10, pp. 81-145, 1935.

[3] K. J. Niklas, "The mechanical significance of clasping leaf sheaths in grasses: evidence from two cultivars of Avena sativa," Annals of Botany, vol. 65, no. 5, pp. 505-512, 1990.

[4] K. J. Niklas, "The mechanical roles of clasping leaf sheaths: evidence from Arundinaria tecta (Poacea) shoots subjected to bending and twisting forces," Annals of Botany, vol. 81, no. 1, pp. 23-34, 1998.

[5] J. Zebrowski, "Complementary patterns of stiffness in stem and leaf sheaths of Triticale-measurements of ultrasound velocity," Planta, vol. 187, no. 3, pp. 301-305, 1992.

[6] L. S. Evans, Z. Kahn-Jetter, C. Marks, and K. R. Harmoney, "Mechanical properties and anatomical components of stems of 42 grass species," Journal of the Torrey Botanical Society, vol. 134, no. 4, pp. 458-467, 2007.

[7] M. J. Crook and A. R. Ennos, "Mechanical differences between free-standing and supported wheat plants, Triticum aestivum L," Annals of Botany, vol. 77, no. 3, pp. 197-202, 1996.

[8] G. H. Dunn and S. M. Dabney, "Modulus of elasticity and moment of inertia of grass hedge stems," Transactions of the American Society of Agricultural Engineers, vol. 39, no. 3, pp. 947-952, 1996.

[9] J. Grace and G. Russell, "The effect of wind on grasses: III. Influence of continuous drought or wind on anatomy and water relations in festuca arundinacea schreb," Journal of Experimental Botany, vol. 28, no. 2, pp. 268-278, 1977.

[10] J. Zebrowski, "Dynamic behaviour of inflorescence-bearing Triticale and Triticum stems," Planta, vol. 207, no. 3, pp. 410-417, 1999.

[11] H. C. Spatz, H. Beismann, F. Brüchert, A. Emanns, and T. Speck, "Biomechanics of the giant reed Arundo donax," Philosophical Transactions of the Royal Society B, vol. 352, no. 1349, pp. 1-10, 1997.

[12] K. Kaack and K. U. Schwarz, "Morphological and mechanical properties of Miscanthus in relation to harvesting, lodging, and growth conditions," Industrial Crops and Products, vol. 14, no. 2, pp. 145-154, 2001.

[13] A. R. Ennos, "The mechanics of the flower stem of the sedge Carex acutiformis," Annals of Botany, vol. 72, no. 2, pp. 123-127, 1993.

[14] S. A. Etnier, "Twisting and bending of biological beams: distribution of biological beams in a stiffness mechanospace," The Biological Bulletin, vol. 205, no. 1, pp. 36-46, 2003.

[15] J. F. V. Vincent, Biomechanics-Materials: A Practical Approach, IRL Press at Oxford University Press, Oxford, UK, 1992.

[16] S. Isnard and N. P. Rowe, "Mechanical role of the leaf sheath in rattans," New Phytologist, vol. 177, no. 3, pp. 643-652, 2008.

[17] R. A. Fischer and M. Stapper, "Lodging effects on high-yielding crops of irrigated semidwarf wheat," Field Crops Research, vol. 17, no. 3-4, pp. 245-258, 1987.

[18] P. M. Berry, M. Sterling, J. H. Spink et al., "Understanding and reducing lodging in cereals," Advances in Agronomy, vol. 84, pp. 217-271, 2004. 

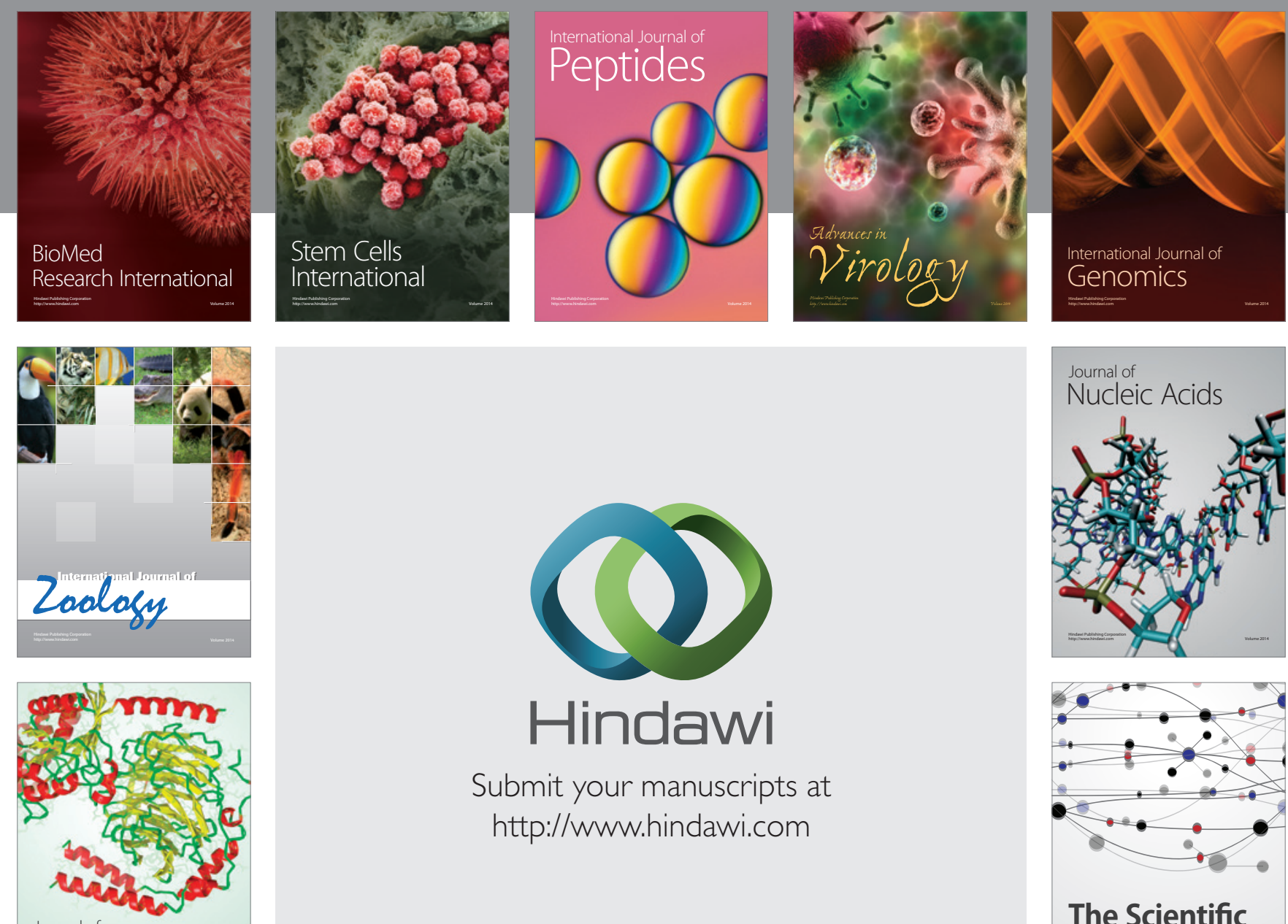

Submit your manuscripts at

http://www.hindawi.com

Journal of
Signal Transduction
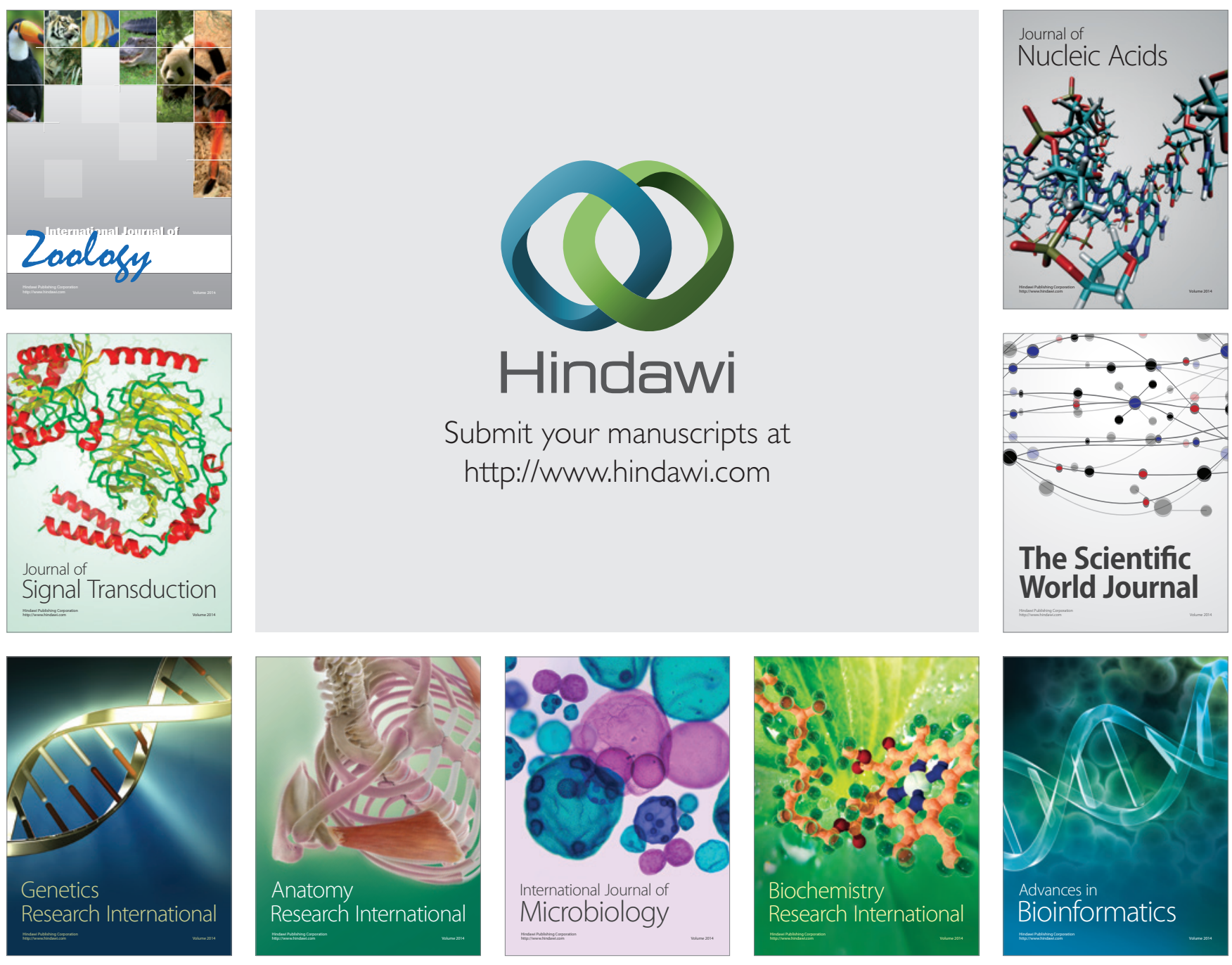

The Scientific World Journal
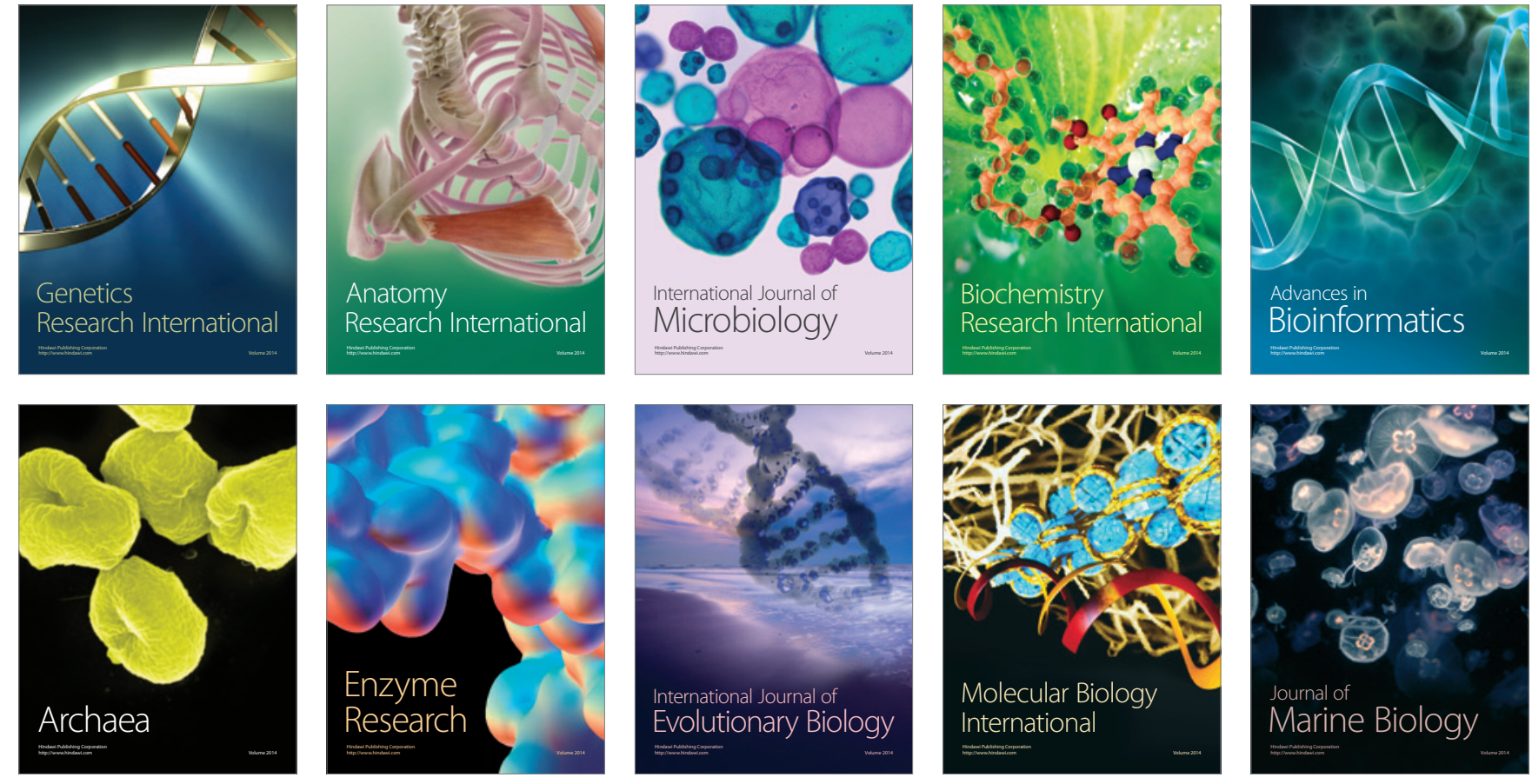